FORMATION Formation emploi

Revue française de sciences sociales

102 | avril-juin 2008

Pêle-mêle

\title{
Scénarios des pratiques de formation post-réforme de mai 2004
}

Vocational training scenarios since the may 2004 reforms

Escenarios de las prácticas de formación posreforma de mayo de 2004

Szenarien betrieblicher Aus- und Weiterbildungspraktiken seit der

Bildungsreform im Mai 2004

Nora Alleki et Catherine de Géry

\section{OpenEdition}

Journals

Édition électronique

URL : http://journals.openedition.org/formationemploi/2248

DOI : 10.4000/formationemploi.2248

ISSN : 2107-0946

Éditeur

La Documentation française

Édition imprimée

Date de publication : 1 juin 2008

Pagination : 55-69

ISSN : 0759-6340

Référence électronique

Nora Alleki et Catherine de Géry, «Scénarios des pratiques de formation post-réforme de mai 2004 », Formation emploi [En ligne], 102 | avril-juin 2008, mis en ligne le 01 juin 2010, consulté le 30 octobre 2020. URL : http://journals.openedition.org/formationemploi/2248; DOI : https://doi.org/10.4000/ formationemploi.2248 


\title{
Entreprise
}

\section{Scénarios des pratiques de formation post-réforme de mai 2004}

Nora Alleki et Catherine de Géry*

\begin{abstract}
Une typologie des pratiques de formation des entreprises en quatre stratégies (offensive, opportuniste, au cas par cas, résistante) permet d'évaluer l'impact de la réforme de la formation continue du 4 mai 2004.
\end{abstract}

Depuis 1971, la formation professionnelle continue (FPC) vise deux objectifs ambivalents : l'adaptation des travailleurs aux évolutions des techniques et l'accès de tous les individus au développement culturel, économique et social. Ce qui se traduit par une division des dispositifs en deux grands ensembles, selon qu'ils s'adressent aux travailleurs qualifiés ou non qualifiés. À partir des années 80 , la FPC accompagne un changement de référence dans la gestion des ressources humaines, qui passe de la qualification attachée au poste de travail à la compétence attachée à l'individu. Elle participe ainsi à la structuration du marché du travail et à l'organisation de l'accès à l'emploi par l'orientation des individus vers le marché interne ou le marché externe du travail ou encore par la mise en attente au sein du dispositif de transition professionnelle. Cet accompagnement se traduit, dans la dernière réforme de la FPC du 4 mai 2004, par le développement d'outils permettant de rendre le salarié acteur de la formation par la co-initiative et la co-responsabilité. Deux ans après la promulgation de la loi, nous nous sommes interrogées sur les effets de cette réforme sur les pratiques de formation des entreprises.
* Nora Alleki est économiste et chargée de mission au ministère du Travail, des Relations sociales, de la Famille et de la Solidarité. Axes de recherche : la relation formationemploi, le développement des compétences et la croissance économique. Elle a publié notamment : N. Alleki \& C. de Géry, "L'impact du modèle de la compétence sur le système de formation professionnelle continue français", Réflexions sur la formation professionnelle et la gestion des compétences. Un état des lieux interdisciplinaire, ouvrage collectif, Éditions Dar El Gharb, Oran, 2007.

Catherine de Géry est économiste et professeur à Advancia-Negocia (CCIP - chambre de commerce et d'industrie de Paris). Axes de recherche : la relation formation-emploi aux niveaux sectoriel et territorial. Elle a notamment publié: C. de Géry, "Réforme du système de formation continue français et modèle de la compétence quelle relation? ", Revue internationale sur le travail et la société, volume 3, n² 2, université de Québec, 2005. C. de Géry, "Les associations de formation professionnelle continue peuvent-elles retrouver un second souffle grâce au développement de la e-formation ? » in G. Rasselet, L'économie sociale en perspective, Presses Universitaires de Reims, 2004. 
Pour répondre à cette question, nous avons, dans un premier temps, identifié et classé les pratiques de formation antérieures à la réforme au travers de la construction d'une typologie des pratiques de formation des entreprises. Dans un second temps, nous avons identifié les mécanismes qui sous-tendent l'évolution des pratiques de formation depuis 1971, à savoir les logiques institutionnelles et le jeu des acteurs. Nous avons alors constaté des régularités et des tendances clés dans l'évolution des pratiques de formation qui nous ont conduit à adopter l'hypothèse de l'inscription du système de formation professionnelle continue, et donc des pratiques de formation, dans un sentier de dépendance. Ce qui nous a permis de construire des scénarios sur les pratiques de formation post-réforme du 4 mai 2004. Ces derniers ont été, dans un troisième temps, testés, à titre exploratoire, dans quatorze entreprises.

\section{UNE TYPOLOGIE DES PRATIQUES DE FORMATION}

Au regard des études décrivant les pratiques de formation des firmes depuis 1971, il a été possible de classer ces dernières en fonction des deux variables discriminantes clés : la segmentation du marché du travail (les pratiques de formation varient selon que la relation salariale s'inscrit dans une configuration de marché interne dominant ou de marché externe dominant) et la nature de l'investissement en formation : stratégique ou obligatoire et subie.

\section{Des pratiques de formation inscrites dans la segmentation du marché du travail}

Les pratiques de formation des firmes sont dépendantes de la conjoncture et donc de l'évolution du marché du travail, fortement marquée par la segmentation du marché en marché interne dominant ou par une configuration de marché externe dominant.

Les théories de la segmentation du marché du travail auxquelles nous adhérons sont initiées par les travaux de Doeringer et Piore (1971) ${ }^{1}$. Pour ces auteurs, la segmentation du marché du travail a pour origine le dualisme de la production qui existe entre la grande entreprise et la PME (petite et moyenne entreprise). La grande entreprise possède un pouvoir de régulation sur la PME en raison de sa capacité à gérer l'incertitude et à s'adapter aux conjonctures défavorables à l'emploi. La grande entreprise exporte l'instabilité de la production en périphérie.

Nous nous référons également à Rubery et Wilkinson (1981) qui développent une approche dynamique et évolutive de la segmentation du marché du travail et regroupent des causes multiples et corrélées de l'existence de cette segmentation (la technologie, la forme de la demande sur le marché des biens, la forme de contrôle et d'organisation du travail, le type d'offre de travail). Puis, nous empruntons à Grimshaw et Rubery (1998) l'idée que les formes de la segmentation ne sont pas stables et que leur taille change selon la conjoncture. Les changements entre différentes formes de marchés internes se comprennent à partir des transformations sur le marché externe (salaire minimum, taux de chômage, système de protection sociale...), des performances de l'entreprise (offre et demande de travail, technologie...) et au niveau des coutumes et des pratiques d'entreprise. Ce qui signifie que les configurations de marché interne dominant et marché externe dominant ne sont pas stables et qu'on peut passer de l'une à l'autre. Ce passage peut s'illustrer par l'analyse de Sengenberger (1981) qui étudie les causes des modifications de la structure de segmentation par les compromis sociaux résultant du pouvoir de négociation des employeurs et des employés. Dans l'approche de la segmentation, l'entreprise est analysée en tant que système ouvert et comme institution productrice de normes ou de règles; ce qui explique que les politiques des firmes dépendent de la conjoncture. Dans une conjoncture favorable à l'emploi, les employeurs auront tendance à cloisonner les marchés internes afin de s'isoler de la pression à la hausse des salaires, alors que les salariés auront la démarche inverse afin de bénéficier de

\footnotetext{
${ }^{1}$ Doeringer et Piore (1971) définissent le marché interne du travail comme une unité administrative dans laquelle la rémunération et l'allocation du travail sont gouvernées par un ensemble de règles et de procédures internes à l'entreprise. À l'opposé, sur les marchés externes du travail, la détermination du salaire, de l'allocation du travail est réalisée par le marché, au sens de la théorie néoclassique.
} 
hausses de salaire. En termes de pratiques de formation, ceci se traduit par un investissement dans les actions de développement de compétences pouvant s'inscrire dans une gestion prévisionnelle des emplois et des compétences. Les actions de formation auront vocation à motiver les salariés et à leur offrir des perspectives de carrière au sein de l'entreprise. La formation n'est plus seulement un outil d'adaptation à l'emploi, mais également un outil de management. En cas de chômage important, nous nous retrouverons dans une approche utilitariste de la formation. Lorsque la conjoncture est défavorable à l'emploi, les entreprises se fixent des objectifs d'ajustements du coût et du volume de l'emploi à la conjoncture, et à court terme. Lorsque la dépense en formation répond à ces objectifs d'ajustements, la formation devient alors un outil de la flexibilité de l'emploi.

Si l'employeur et le salarié décident de coopérer, ce qui reste probable dans la configuration de marché interne dominant, une stratégie de donnant-donnant devient possible. La coopération joue donc en faveur de chacun des acteurs qui recherche une rentabilisation des investissements en formation et de prévention des risques. S'il n'y a aucune procédure de concertation, ce qui reste probable dans la configuration de marché externe dominant, chacun opte pour une stratégie de résistance dans laquelle l'effort et la rémunération sont au minimum.

\section{Des pratiques de formation entre investissement stratégique et investissement obligatoire}

Les bilans empiriques de la formation professionnelle continue du Céreq permettent de distinguer deux grandes politiques de formation, selon que les entreprises investissent stratégiquement en formation ou à hauteur de l'obligation légale. La taille et le secteur d'activité de la firme déterminent le type d'investissement. Ces deux effets agissent simultanément.

On a ainsi, d'une part, l'entreprise de grande taille ou la PME, appartenant à une branche d'activité innovante ou organisée en réseau, qui investira stratégiquement en formation, appelée entreprise formatrice. Nous parlerons alors de stratégies de formation et non de pratiques de formation concernant ces entre- prises (Alleki, 2000). Ce type de firme investit dans la formation générale et dans des structures internes spécifiquement destinées à la formation. Toutefois, consciente du risque de non-retour en investissement, l'entreprise formatrice n'investira pas dans la formation générale sans se prémunir contre ce risque. En général, elle parvient à le minimiser en mobilisant les outils du co-investissement en formation (capital temps formation, formation en dehors du temps de travail, certification...) ou le soutien financier de l'État.

D'autre part, on a l'entreprise qui investira dans la formation à hauteur de l'obligation légale. Cette dernière a plutôt un comportement proche de ce que G. Becker (1964) décrit dans son modèle. L'entreprise souhaite optimiser son budget de formation et pour cela elle a intérêt à investir uniquement dans les actions de formation spécifiques pour lesquelles le risque de non-retour sur investissement est minimisé. La formation générale n'est donc circonscrite qu'à des cas particuliers.

\section{Des pratiques de formation classées en quatre grands ensembles}

Le croisement des deux critères discriminants, la nature de l'investissement en formation et l'inscription des pratiques de formation dans une configuration de marché interne dominant ou de marché externe dominant permettent de classer les pratiques de formation antérieures à la réforme en quatre ensembles (tableau 1) (Alleki \& Géry, 2006). Pour chaque configuration de marché, on peut anticiper un niveau de participation à la formation, un type d'élaboration du plan de formation, des types de formation et un mode de gestion de la formation. 2 Dans les modèles de G. Becker, l'accumulation de connais-
sances obéit à un comportement de maximisation sous une dou-
ble contrainte à la fois de gains et de coûts (directs et
''opportunité), dans un cadre de concurrence pure et parfaite
dans lequel les facteurs de production sont substituables. La for-
mation est ainsi un processus d'acquisition de connaissances
coûteux, qui peut toutefois engendrer à terme des revenus
additionnels. 
Tableau 1

Typologie des pratiques de formation avant la réforme du 4 mai 2004

\begin{tabular}{|c|c|c|}
\hline & Logique d'investissement stratégique & Logique d'investissement obligatoire \\
\hline $\begin{array}{l}\text { Marché } \\
\text { interne } \\
\text { dominant }\end{array}$ & $\begin{array}{l}\text { Pratique offensive } \\
\text { - Le taux de participation financière à la forma- } \\
\text { tion est élevé (supérieur à } 5 \% \text { de la masse sala- } \\
\text { riale brute). } \\
\text { - L'investissement en formation résulte d'un } \\
\text { compromis salarial. } \\
\text { - L'ensemble des outils pour développer la co- } \\
\text { initiative des salariés à la formation est utilisé. } \\
\text { - Il y a un développement de l'accompagne- } \\
\text { ment individualisé de la formation et une garantie } \\
\text { collective de cette politique, notamment par la } \\
\text { certification et la validation des acquis de l'expé- } \\
\text { rience (VAE). }\end{array}$ & $\begin{array}{l}\text { Pratique opportuniste } \\
\text { - Le taux de participation est proche du mini- } \\
\text { mum légal (autour de } 1,6 \text { à } 2 \% \text { de la masse } \\
\text { salariale brute). } \\
\text { - La politique de formation vise l'adaptation à } \\
\text { l'emploi et la flexibilité du volume de travail et } \\
\text { des rémunérations. } \\
\text { - Le financement de la formation est négocié de } \\
\text { gré à gré. L'entreprise souhaite développer les } \\
\text { compétences et véhiculer des valeurs fondées } \\
\text { essentiellement sur la reconnaissance des résultats } \\
\text { ainsi que sur l'effort et le mérite au travail. La for- } \\
\text { mation est un moyen de récompenser certaines } \\
\text { catégories de salariés. }\end{array}$ \\
\hline $\begin{array}{l}\text { Marché } \\
\text { externe } \\
\text { dominant }\end{array}$ & $\begin{array}{l}\text { Pratique du cas par cas } \\
\text { - Le taux de participation financière est proche } \\
\text { de la moyenne nationale (autour de } 2,5 \text { à } 3 \% \text { ). } \\
\text { - La politique de formation ne suit pas un plan } \\
\text { prédéterminé en fonction d'une cohérence } \\
\text { d'ensemble. } \\
\text { - La gestion de la formation se fait au cas par } \\
\text { cas en fonction de la rentabilité immédiate qu'elle } \\
\text { procure et des règles de droit. Le cas par cas } \\
\text { contient un risque élevé de renforcer les inégalités } \\
\text { d'accès à la formation et un sous-investissement } \\
\text { en formation. } \\
\text { - Malgré les risques élevés de non-retour sur } \\
\text { investissement, étant donné l'absence de pers- } \\
\text { pectives et d'objectifs clairement définis et } \\
\text { acceptés par les salariés, l'employeur investit } \\
\text { stratégiquement dans la formation dans le but } \\
\text { de sélectionner et de développer les compé- } \\
\text { tences clés. Les salariés qui voient leurs } \\
\text { demandes satisfaites vont quant à eux souhaiter } \\
\text { d'abord se constituer un capital de compé- } \\
\text { tences, garant de leur employabilité future. }\end{array}$ & $\begin{array}{l}\text { Pratique de résistance } \\
\text { - Le taux de participation financier est proche du } \\
\text { minimum légal. } \\
\text { - Le plan de formation vise les gains de producti- } \\
\text { vité et la réduction du coût salarial. } \\
\text { - Les formations financées par les entreprises } \\
\text { sont principalement d'adaptation au poste et } \\
\text { prennent, pour la plupart, la forme d'apprentis- } \\
\text { sage par la pratique. } \\
\text { - Toutes les possibilités de déduction de charges } \\
\text { dans le plan de formation sont utilisées (dépenses } \\
\text { de personnel en lien avec la préparation du plan } \\
\text { de formation, salles de formation, tutorat, certifi- } \\
\text { cation, amortissement des équipements utilisés en } \\
\text { formation). La logique est ici comptable. } \\
\text { - La gestion des ressources humaines est vue à } \\
\text { court terme. Les travailleurs sont considérés res- } \\
\text { ponsables de l'évolution de leurs compétences. }\end{array}$ \\
\hline
\end{tabular}




\section{LES SCÉNARIOS DES PRATIQUES DE FORMATION}

\section{L'inscription des pratiques \\ de formation dans un sentier de dépendance}

Le système de FPC français est singulier par rapport aux systèmes de formation d'autres pays. Il se caractérise par une obligation légale de financement de la formation, une mutualisation des fonds auprès d'organismes collecteurs indépendants, une régulation des besoins et des pratiques par des négociations de nature tripartite et par un ensemble d'acteurs hétérogènes aux statuts juridiques variés. Ces singularités correspondent aux spécificités sociétales suivantes :

- L’État a une place importante et ancienne dans la régulation de la formation des individus.

- L'État occupe une place centrale dans une négociation en matière de formation, de nature tripartite, si bien qu'il contrôle, pilote et impulse les négociations.

- Le niveau privilégié de la négociation dans le domaine de la formation est la branche professionnelle. Elle joue un rôle de régulation sociale en gérant la concurrence et les flux des qualifications et des emplois entre les entreprises d'une même branche ou entre les branches. La branche est un acteur essentiel en matière d'organisation de la transférabilité des compétences (Tallard, 2004). Elle négocie par exemple les priorités attendues de la formation en termes de développement des compétences, d'adaptation ou de maintien dans l'emploi.

- Le système éducatif français est enraciné dans la noblesse et les relations professionnelles régulées par la logique de l'honneur (P. d'Iribarne, 1989). La noblesse se traduit par une hiérarchisation des filières de formation et des métiers en faveur des formations d'enseignement général et au détriment des formations professionnelles. Et la logique de l'honneur explique dans quelle mesure la conquête sociale est le fruit de la rupture ou de la lutte salariale et non pas de compromis négociés.

Ces particularités du système français, associées à un jeu d'acteurs complexe, impliquent des pratiques spécifiques des firmes qui, entre 1971 et 2000, sont classables en trois périodes (Alleki, 2000) ${ }^{3}$ :

1. Pendant les années 70, les entreprises remplissent l'obligation légale sans considérer l'ensemble des opportunités liées au budget de formation.

2. Pendant les années 80 , la formation devient un investissement pour adapter les qualifications aux évolutions techniques. Ce qui se traduit par une augmentation des fonds pour la formation, du nombre de stagiaires et par le développement d'outils et de structures internes destinés à la formation.

3. Pendant les années 90 , les entreprises cherchent à stabiliser leur budget de formation et certaines, les entreprises formatrices (entreprises de grande taille et PME appartenant aux branches d'activité innovantes ou organisées en réseau) commencent à considérer, l'investissement en formation comme une stratégie. La formation devient à la fois un instrument pour améliorer l'employabilité des salariés et un outil au service de la flexibilité des entreprises.

4. Les années 2000, quant à elles, sont principalement caractérisées par le développement de l'entreprise formatrice, qui investit stratégiquement en formation. La conception de la formation en tant qu'investissement consiste à détourner provisoirement du temps de travail de l'activité de production et donc à renoncer à une production immédiate ; il s'agit de constituer un capital de compétences, inséparable du travailleur qui décide de l'exploiter ou de l'enrichir.

L'évolution des pratiques de formation émanant de la loi fondatrice de 1971 montre qu'à chaque nouvelle loi, des éléments de continuité et des régularités peuvent être identifiés. En effet, les pratiques de formation des firmes sont dépendantes des régulations publiques, du changement technique et des spécificités sociétales françaises (Maurice, Sellier et Sylvestre, 1982).

\footnotetext{
${ }^{3}$ Cette classification s'est construite à partir des résultats des bilans de la formation (exploitations des déclarations fiscales $n^{\circ} 24 / 83$ entre 1971 et nos jours établies par le Céreq), à l'aide d'indicateurs suivants :

1. le taux de participation financière (TPF) qui correspond aux dépenses de formation rapportées à la masse salariale brute ;

2. le taux de stagiaires qui est le nombre de stagiaires rapporté au nombre de salariés.

La durée moyenne des stages est égale au nombre d'heures de formation rapporté au nombre de stagiaires.
} 


\section{Encadré 1 \\ Divers dispositifs de formation}

Le plan de formation : mis en place dans les entreprises depuis la loi Delors de juillet 1971, ce dispositif est défini dans le Code du travail depuis la loi du 4 mai 2004. II rassemble l'ensemble des actions de formation définies dans le cadre de la politique de gestion du personnel de l'entreprise. II peut également prévoir des actions de bilan de compétences et de validation des acquis de l'expérience et proposer des formations qui participent à la lutte contre l'illettrisme. l'élaboration du plan de formation est sous la responsabilité pleine et entière du chef d'entreprise, après consultation des représentants du personnel. Le plan de formation peut comporter trois types d'actions visant l'adaptation du salarié au poste de travail : respectivement actions liées à l'évolution des emplois ou participant au maintien dans l'emploi ou ayant pour objet le développement des compétences des salariés.

Le congé individuel de formation (CIF), institué par la loi Delors de juillet 1971, est un droit permettant, sous certaines conditions, à tout salarié de suivre, à son initiative et à titre individuel, une formation de son choix, tout au long de sa vie professionnelle, afin « d'accéder à un niveau supérieur de qualification, de changer d'activité ou de profession et de s'ouvrir plus largement à la culture et à la vie sociale » selon le livre IX du Code du travail. Pour en bénéficier, le salarié doit présenter sa demande à l'employeur, selon une procédure déterminée. Le salarié peut bénéficier d'une prise en charge de sa rémunération et des frais liés au congé par l'organisme paritaire collecteur agréé au titre du CIF (OPACIF) ou encore d'organismes dont la compétence est limitée à une entreprise ou un groupe d'entreprises (AGECIF).

Les Engagements de développement de la formation (EDDF), mis en place en 1984, concernent les entreprises qui s'engagent, sur plusieurs années au-delà de leur obligation légale, et dont les projets de formation s'inscrivent dans une réflexion stratégique de développement. L'accent est mis sur la reconnaissance et la transférabilité des qualifications, avec une attention portée aux très petites entreprises.

Les EDDF sont conclus dans le cadre d'un accord national de branche au plan professionnel ou interprofessionnel ou d'un accord bilatéral entre le Préfet de la région et une entreprise.

l'État participe à condition que l'entreprise réalise une augmentation significative de son investissement en formation (net de subventions).

L'entreprise aidée au titre de l'EDDF peut bénéficier d'un cofinancement du Fonds social européen. A contrario, elle ne peut cumuler l'aide EDDF avec une contribution financière du Fonds national pour I'emploi (FNE Formation...).

Le droit individuel à la formation (DIF), instauré par la loi du 4 mai 2004, est un crédit de 20 heures de formation par an (pour un temps complet et au prorata du temps de travail pour un temps partiel) cumulables sur six ans. En fonction de ce que l'accord collectif prévoit, le temps de formation se déroule en partie ou entièrement en dehors du temps de travail pour des actions dites de développement des compétences ou de maintien dans l'emploi. Ce droit s'adresse au salarié en contrat à durée indéterminée ayant au moins un an d'ancienneté et au salarié en contrat à durée déterminée ayant acquis au moins quatre mois d'ancienneté dans l'entreprise au cours des 12 derniers mois. Ce droit est transférable en cas de licenciement, à l'exception du licenciement individuel pour faute lourde et dans d'autres cas si un accord collectif le prévoit. Si le DIF est à l'initiative du salarié, l'employeur conserve le pouvoir d'accepter ou de refuser ce droit. Même si la loi prévoit une sanction lorsque l'entreprise refuse plus de deux fois et un recours au salarié pour faire valoir son DIF, le faible degré de contrainte ne garantit pas l'aboutissement du projet du salarié. Par ailleurs, même si les procédures qui entourent le plan de formation, notamment les consultations des représentants du personnel, sont renforcées et que leur non-respect est financièrement sanctionné, l'employeur n'est pas obligé de proposer des formations contribuant au 


\section{Encadré 1 (suite)}

développement de compétences. A contrario, si un salarié refuse de suivre une formation dans le temps de travail, alors que les charges sont assumées par l'employeur (hors VAE et bilans de compétences), ceci constitue une faute qui peut justifier le licenciement.

Le dispositif des périodes de professionnalisation a été créé par la loi du 4 mai 2004 « relative à la formation professionnelle tout au long de la vie et au dialogue social ». Ces périodes ont pour objet de favoriser, par des actions de formation alternant enseignements théoriques et pratiques, le maintien dans l'emploi des salariés en contrat à durée indéterminée, notamment ceux qui comptent 20 ans d'activité professionnelle ou qui sont âgés d'au moins 45 ans et disposent d'une ancienneté minimum d'un an dans leur dernière entreprise.

Le contrat de professionnalisation, institué par la loi du 4 mai 2004, s'adresse à tous les jeunes âgés de 16 à 25 ans et aux demandeurs d'emploi âgés de 26 ans et plus. Ce dispositif remplace les contrats d'adaptation, d'orientation et de qualification qui existaient depuis 1984. Son objectif est de leur permettre d'acquérir une qualification professionnelle et de favoriser leur insertion ou réinsertion professionnelle. Les bénéficiaires âgés de 16 à 25 ans révolus sont rémunérés en pourcentage du Smic (salaire minimum interprofessionnel de croissance) selon leur âge et leur niveau de formation, les salariés âgés de 26 ans et plus perçoivent une rémunération qui ne peut être ni inférieure au Smic ni à 85 \% du salaire minimum conventionnel. Ce contrat ouvre droit pour l'employeur, pour certaines embauches et dans certaines limites, à une exonération de cotisations patronales de sécurité sociale.

La Gestion prévisionnelle des emplois et des compétences (GPEC) est une gestion anticipative des ressources humaines, en fonction des contraintes de l'environnement et des stratégies de l'entreprise. Cette forme de gestion est promue par l'État à partir de la seconde moitié des années 90. En 2002, l'État institue deux types de conventions: d'appui à l'élaboration de plan de GPEC, de sensibilisation aux enjeux de la GPEC. La loi de Programmation pour la Cohésion sociale du 18 janvier 2005, dite Loi Borloo lloi $n^{\circ} 2005-321$, a instauré une obligation de négociation triennale sur la Gestion prévisionnelle de l'emploi et des compétences. Ainsi, un dispositif de GPEC s'appuie sur :

- un volet collectif pour détecter en amont les questions relatives à l'évolution des métiers, des emplois, des compétences, des effectifs et anticiper les risques d'écarts entre besoins et ressources sur un plan qualitatif et quantitatif, et définir des règles et moyens facilitant l'ajustement;

- un volet individuel pour promouvoir de manière anticipée le développement des capacités d'évolution et de l'employabilité de chaque salarié dans le cadre de son parcours professionnel.

Ce qui nous amène à poser l'hypothèse que le système de FPC, et par conséquent les pratiques de formation des firmes sont situées dans un sentier de dépendance, concept emprunté à la théorie évolutionniste (Nelson \& Winter, 1982). Le sentier de dépendance (path dependancy) postule que les performances et les trajectoires des firmes dépendent largement de leur histoire particulière et des routines qu'elles ont accumulées. Appliqué à la formation, cela signifie que l'environnement institutionnel affecte le choix des entreprises, choix reproduits au sein des firmes par un processus d'apprentissage et de sélection de routines. Certaines de ces routines organisationnelles sont tacites et difficilement transférables, comme le choix des organismes de formation, la sélection des salariés, les manières d'intégrer les réformes. Ainsi, un choix effectué en un temps donné va conditionner le développement futur d'un certain nombre de routines organisationnelles et donc des choix futurs de l'entreprise. 
À partir d'une typologie des pratiques de formation (partie 1) et au regard des accords de branches signés après la loi du 4 mai 2004, en adoptant cette hypothèse de sentier de dépendance, il nous est possible de construire des scénarios des pratiques de formation post-réforme (voir tableau 2). Pour chacun de ces scénarios, nous verrons comment évoluent :

- le montant du budget consacré à la formation ;
- l'horizon temporel de la gestion de la formation : court, moyen ou long terme ;

- la nature des formations privilégiées : formation générale ou spécifique ;

- les modalités de fonctionnement du droit individuel de formation (en temps de travail, hors temps de travail, information, possibilité de cumul).

Tableau 2

Typologie des scénarios de formation post-réforme du 4 mai 2004

\begin{tabular}{|c|c|c|}
\hline & Logique d'investissement stratégique & Logique d'investissement obligatoire \\
\hline $\begin{array}{l}\text { Marché } \\
\text { interne } \\
\text { dominant }\end{array}$ & $\begin{array}{l}\text { Scénario offensif } \\
\text { - Il y a une recherche d'optimisation des finance- } \\
\text { ments pour contenir une augmentation du budget } \\
\text { de formation. Ce qui signifie qu'une partie du plan } \\
\text { de formation sera "diffable "; le droit individuel à } \\
\text { la formation (DIF) est intégré en partie au plan de } \\
\text { formation et mis en œeuvre sur temps de travail. } \\
\text { - La gestion prévisionnelle de la formation } \\
\text { s'accentue avec l'utilisation des nouveaux outils tels } \\
\text { que les plans pluriannuels et la possibilité d'utiliser } \\
\text { les } 120 \text { heures cumulées du DIF par anticipation. } \\
\text { Ce cumul par anticipation est utilisé en contrepartie } \\
\text { de formations longues et diplômantes. } \\
\text { - Le DIF hors temps de travail est possible pour } \\
\text { des formations générales transférables de courte } \\
\text { durée qui améliorent la polyvalence des salariés } \\
\text { (par exemple, les formations de spécialisation en } \\
\text { langues, informatique, conduite d'entretiens, } \\
\text { coaching...) ou qui permettent d'intégrer les sala- } \\
\text { riés aux objectifs de la firme (par exemple la } \\
\text { validation des acquis de l'expérience (VAE) à } \\
\text { destination des ouvriers). } \\
\text { - La politique de communication interne accom- } \\
\text { pagne la politique de formation. }\end{array}$ & $\begin{array}{l}\text { Scénario opportuniste } \\
\text { - L'entreprise recherche un budget constant. } \\
\text { - Il n'y a pas de gestion prévisionnelle de la for- } \\
\text { mation et l'initiative du salarié est réduite par } \\
\text { celle de l'employeur. } \\
\text { - L'entreprise investit dans les formations spéci- } \\
\text { fiques et limite son investissement dans les forma- } \\
\text { tions générales en faisant prendre en charge par } \\
\text { le salarié ou l'État le poids de cet investissement. } \\
\text { - Le DIF hors temps de travail n'est accepté par } \\
\text { l'employeur qu'en complément des actions ins- } \\
\text { crites dans l'axe " développement des compé- } \\
\text { tences » du plan de formation. Ces formations } \\
\text { seront acceptées par les salariés tant qu'elles res- } \\
\text { teront sanctionnées par un diplôme ou titre profes- } \\
\text { sionnel ou parce qu'elles offrent des perspectives } \\
\text { d'évolution et/ou de progression salariale. } \\
\text { - Les possibilités de formations générales en hors } \\
\text { temps de travail seront renvoyées vers le congé } \\
\text { individuel de formation si le salarié ne réussit pas } \\
\text { à convaincre l'employeur de son intérêt. } \\
\text { - L'information sur la réforme est plutôt indivi- } \\
\text { dualisée. }\end{array}$ \\
\hline $\begin{array}{l}\text { Marché } \\
\text { externe } \\
\text { dominant }\end{array}$ & $\begin{array}{l}\text { Scénario du cas par cas } \\
\text { - Le budget de formation reste constant. } \\
\text { - II n'y a pas de gestion prévisionnelle de la for- } \\
\text { mation. L'entreprise est en position d'attente et } \\
\text { examine les réactions des concurrents avant de } \\
\text { définir la stratégie de formation. } \\
\text { - Le DIF hors temps de travail n'est pas intégré au } \\
\text { plan de formation. Le DIF peut être un bon outil } \\
\text { pour repérer les salariés motivés ou intégrés aux } \\
\text { objectifs de la firme. L'entreprise va donc gérer au } \\
\text { fur et à mesure et au cas par cas les demandes de } \\
\text { DIF. Les demandes examinées sont celles des sala- } \\
\text { riés qualifiés et informés, au détriment des autres. }\end{array}$ & $\begin{array}{l}\text { Scénario de résistance } \\
\text { - L'entreprise maintient le budget au minimum. } \\
\text { - Il n'y a pas de gestion prévisionnelle de la } \\
\text { formation. } \\
\text { - L'entreprise investit dans les formations spéci- } \\
\text { fiques et refuse de prendre en charge des forma- } \\
\text { tions générales. } \\
\text { - Le DIF est exceptionnel pour les plus qualifiés } \\
\text { hors temps de travail } \\
\text { - La communication sur la politique de formation } \\
\text { est quasi inexistante. }\end{array}$ \\
\hline
\end{tabular}




\section{Quatre scénarios sur les pratiques de formation post-réforme}

Ces scénarios mettent en avant les quatre grandes tendances suivantes :

1. Les entreprises cherchent à maintenir leur budget car le financement du système de formation est parvenu à maturité ;

2. Les exigences de flexibilité incitent les entreprises à développer une gestion prévisionnelle de la formation ;

3. Les entreprises tentent de transférer et de partager les risques de non-retour sur investissement en développant les outils de co-investissement en formation ;

4. La réforme du 4 mai 2004, et particulièrement la création du droit individuel à la formation, implique de la part des entreprises une politique de communication interne spécifique.

À titre exploratoire, ces tendances seront testées dans quatorze entreprises. Toutefois, une attention particulière sera accordée au DIF, pour plusieurs raisons. Ce dispositif est novateur du fait de l'attachement à l'individu d'un droit de formation et de son possible exercice en dehors du temps de travail. Largement médiatisé et suscitant des attentes des responsables de formation, ce nouveau dispositif a été généralement le premier à être investi par les entreprises. Toutefois, il a suscité de nombreuses réserves sur les capacités de tous les salariés à devenir acteurs, et notamment à user de ce nouveau droit (Maggi-Germain, 2004). Nous avons alors formulé quatre hypothèses sur la mise en œuvre du DIF :

- H1 : Le DIF sera principalement articulé au plan de formation, étant donné qu'aucun financement spécifique du DIF n'est prévu et que les entreprises n'envisagent pas d'augmenter leur budget de formation ;

- H2 : La réforme devrait impulser le développement d'une gestion pluriannuelle de la formation s'appuyant sur une optimisation de l'articulation de l'ensemble des outils ${ }^{4}$;

\footnotetext{
${ }^{4}$ La gestion des DIF ne pourra pas se faire uniquement par une relation bilatérale, comme l'analyse la théorie du capital humain, mais par une approche organisationnelle de la FPC (Hanchane \& Stankiewicz, 2004). Cette organisation peut se traduire par une gestion prévisionnelle permettant de lisser les besoins de formation et, en s'appuyant sur la possibilité de cumul du DIF sur 6 ans, de financer des formations de plus longue durée.
}

- H3 : Le DIF sera réalisé principalement pendant le temps de travail sauf pour trois catégories de salariés :

- ceux sur travail posté 5 pour des questions de coûts ${ }^{6}$;

- ceux qualifiés ou à haut potentiel que l'entreprise souhaite garder ;

- ceux avec qui l'entreprise souhaite développer le co-investissement en formation, soit pour les intégrer aux objectifs de la firme, soit pour diminuer le risque de non-retour sur investissement.

- H4 : L'accès au DIF dépend à la fois de l'appétence des salariés à la formation et de l'accès à l'information sur le dispositif directement lié à la politique de communication interne.

\section{LA MISE À L'ÉPREUVE DES SCÉNARIOS DANS QUATORZE ENTREPRISES}

Nous avons mené des entretiens auprès de treize entreprises issues de branches d'activité couvrant 188153 salariés et d'une entreprise d'intérim ayant employé 552650 intérimaires (voir tableau 3) ${ }^{7}$.

\footnotetext{
${ }^{5}$ Le travail posté est défini par des critères qui sont la continuité, le type de rotation et l'alternance des équipes. Par extension, nous prendrons en compte les salariés au contact du client final dans le travail posté.

${ }^{6}$ Un travailleur posté, s'il est formé sur son temps de travail, doit être remplacé, ce qui représente un doublement de son salaire en termes de coût alors que s'il est formé en dehors de son temps de travail, le coût est de 1,5 fois son salaire.

${ }^{7}$ Cette enquête a été réalisée au moyen d'entretiens téléphoniques d'une durée moyenne d'une heure, réalisés entre juillet et août 2006 auprès des responsables formation, dans le cadre d'un partenariat recherche entre le GARF (Groupement des acteurs et responsables de formation), l'OFEM (Observatoire de la formation, de l'emploi et des métiers) de la chambre de commerce et d'industrie de Paris et NEGOCIA, une école de la chambre de commerce et d'industrie de Paris. Les principaux thèmes abordés concernent la perception de la réforme, le plan de formation, le budget de formation, le DIF, les périodes de professionnalisation et les contrats de professionnalisation, la politique de communication sur la réforme auprès des salariés.
} 
Tableau 3

Profils socio-économiques des entreprises

\begin{tabular}{|c|c|c|}
\hline Secteur & Effectif & Taux de participation financière 2005 en $\%\left({ }^{*}\right)$ \\
\hline Banque & 1200 & 4 \\
\hline Transport aérien & 56600 & 8,70 \\
\hline Gaz & 110000 & 5 \\
\hline Équipementiers & 18 & 4 \\
\hline Métallurgie & 620 & «Supérieure au seuil » \\
\hline Travaux publics & 4500 & 3,30 \\
\hline $\begin{array}{l}\text { Commerce de détail } \\
\text { alimentaire }\end{array}$ & 2188 & 3,80 \\
\hline $\begin{array}{l}\text { Commerce de détail } \\
\text { spécialisé }\end{array}$ & 8905 & 2,40 \\
\hline Commerce de gros & 449 & 3,80 \\
\hline Organisme de formation & 25 & 2,50 \\
\hline Prestations de service & 148 & 3,10 \\
\hline Intérim & 552650 intérimaires & 2,10 \\
\hline Tourisme social & $\begin{array}{c}1500 \\
\text { en temps plein }\end{array}$ & 2,40 \\
\hline Habillement & 2000 & 2,80 \\
\hline
\end{tabular}

$(*)$ : Le Code du travail impose aux entreprises des contributions financières minimales calculées sur leur masse salariale. Celles-ci sont, soit constituées de dépenses de l'entreprise, soit constituées de versements à des organismes spécialisés, créés et gérés par les partenaires sociaux : les Organismes paritaires collecteurs agréés (OPCA). Pour ces entreprises de plus de 20 salariés, le taux de participation financière obligatoire est de $1,6 \%$ de la masse salariale brute se répartissant ainsi :

- $0,20 \%$ destiné au financement des congés de formation ;

- $0,50 \%$ destiné au financement des contrats et périodes de professionnalisation et du droit individuel à la formation ;

- le solde $(0,90 \%)$ étant affecté au financement des actions de formation continue des salariés de l'entreprise.

Note : Les entreprises ne sont pas représentatives de l'échantillon de leur secteur, notamment en ce qui concerne la variation de taille ; l'appartenance sectorielle est donc fournie à titre de variable illustrative et non pas explicative.

\section{Le scénario offensif : des opportunités insuffisamment exploitées}

Le scénario offensif semble être adopté par trois entreprises du secteur bancaire, du transport aérien et du secteur du gaz. Elles ont peu investi les possibilités offertes par la réforme et restent marquées par leur stratégie antérieure. Leurs taux de participation financière (TPF) se situent entre 4 et 8,7\%. Elles ne s'attendent pas à un changement d'attitude des salariés à court terme, mais éventuellement à long terme. Toutefois, elles reconnaissent que la réforme peut rendre le salarié acteur.

L'entreprise du gaz n'exploite pas la possibilité de cumul du DIF sur six ans et sa gestion de la formation est annuelle. En revanche, les firmes du secteur aérien et de la banque, ayant une gestion sur trois ans, utilisent le cumul des 120 heures du DIF pour respectivement toutes les catégories de salariés et pour les salariés les plus qualifiés. Le budget formation est susceptible d'augmenter dans le transport aérien et la banque, non pas pour satisfaire des demandes nouvelles des salariés qui seraient liées à la réforme, mais pour se conformer à l'obligation d'application de normes de formation dans l'exercice de certains de leurs métiers. Toutes ces entreprises ont lancé une campagne de communication interne pour informer les salariés. Les demandes de DIF peuvent être formulées à tout moment par écrit auprès du responsable formation. La réalisation des DIF se fait pendant le temps de travail dans l'entreprise du gaz, hors temps de travail dans le transport aérien, à l'exception du 
Tableau 4

Profils socio-économiques des entreprises du scénario offensif

\begin{tabular}{|c|c|c|c|}
\hline Branches & $\begin{array}{l}\text { Date de début des demandes } \\
\text { (durée de mise en œuvre } \\
\text { à la date de l'entretien) }\end{array}$ & $\begin{array}{l}\text { Nombres de } \\
\text { demandes émises } \\
\text { (\% de salariés) }\end{array}$ & Réponse \\
\hline Banque & 8 juillet 2005 ( 12 mois) & $\begin{array}{c}11 \text { en diplômant et } 2 \text { normales } \\
(1 \%)\end{array}$ & positive \\
\hline $\begin{array}{l}\text { Transport } \\
\text { aérien }\end{array}$ & 7 mai 2005 (14 mois) & $\begin{array}{c}800 \\
(1,4 \%) \\
\end{array}$ & positive \\
\hline Gaz & 1er janvier 2006 (6 mois) & $\begin{array}{l}2000 \\
(1,8 \%)\end{array}$ & $\begin{array}{l}\text { accord de branche, } \\
\text { coeur de métier }\end{array}$ \\
\hline
\end{tabular}

Source : Nora Alleki, Catherine de Géry, administration du questionnaire sur les pratiques de formation, juillet-août 2006.

personnel navigant, et dans et hors temps de travail dans l'entreprise de la banque.

\section{Le cas par cas : une appropriation} des nouveaux outils par tâtonnement

Ce scénario semble être adopté par l'entreprise de la métallurgie (620 salariés), des travaux publics (4 500 salariés) et des équipementiers (18 salariés). Leurs taux de participation financière à la formation sont dans la moyenne de leurs branches professionnelles. La durée moyenne des stages varie de deux à trois jours pour les entreprises de la métallurgie et des travaux publics à six jours pour l'entreprise de la branche de l'équipement. Ces entreprises ne s'attendent pas à un changement d'attitude des salariés ni à un changement dans les pratiques de formation. Seule l'entreprise des travaux publics permet le cumul anticipé du DIF sur six ans pour les formations diplômantes et annonce une gestion tri- annuelle de la formation. Selon le responsable formation de l'entreprise des travaux publics, le budget de formation, qu'il nomme "esobudget», ne devrait pas augmenter. Le financement du DIF est prévu sur le plan de formation, en rendant celuici «diffable». Les salariés sont informés du contenu général de la réforme par la commission formation des comités d'entreprise. L'entreprise mise sur une information sur les dispositifs plus précise, individualisée, au travers de l'entretien professionnel ou de supports accompagnant le bulletin de salaire. Les demandes de DIF sont formulées par écrit et soumises au responsable de la formation. La gestion du DIF est prévue pendant le temps de travail pour l'entreprise de la métallurgie et des travaux publics, sauf pour les actions de développement des compétences, et hors temps de travail pour l'entreprise de l'équipement. Seule l'entreprise de travaux publics a exploré le dispositif des périodes de professionnalisation.

Tableau 5

Profils socio-économiques des entreprises du scénario du cas par cas

\begin{tabular}{|l|c|c|c|}
\hline \multicolumn{1}{|c|}{ Branches } & $\begin{array}{c}\text { Date de début des demandes } \\
\text { (durée de mise en œuvre } \\
\text { à la date de l'entretien) }\end{array}$ & $\begin{array}{c}\text { Nombres de demandes } \\
\text { émises } \\
\text { (\% de salariés) }\end{array}$ & Réponses \\
\hline Équipementiers & ler avril 2006 (3 mois) & $\begin{array}{c}0 \\
(0 \%)\end{array}$ & positive \\
\hline Métallurgie & ler janvier 2005 (18 mois) & $\begin{array}{c}6 \\
(1 \%)\end{array}$ & positive \\
\hline Travaux publics & 4 mai 2004 (26 mois) & $\begin{array}{c}50 \\
(1,1 \%)\end{array}$ \\
\hline
\end{tabular}

Source : Nora Alleki, Catherine de Géry, administration du questionnaire sur les pratiques de formation, juillet-août 2006. 


\section{Le scénario opportuniste : une vision} utilitariste de la réforme

Sept des entreprises questionnées semblent adopter le scénario opportuniste dans les branches du commerce de gros non alimentaire (449 salariés), du commerce de détail et de gros à prédominance alimentaire (2 188 salariés), du commerce de détail spécialisé (8 905 salariés), de la formation (25 salariés), de la prestation de services rattachée à la branche automobile (148 salariés), du tourisme social (1 500 tempsplein et 3000 saisonniers) et de l'intérim (552 650 intérimaires). Elles adoptent une réflexion poussée sur les opportunités de la réforme lorsqu'elles sont en phase de professionnalisation de leurs salariés, tout en cherchant à maintenir les coûts de formation, par exemple dans le commerce. Ces entreprises ont un taux de participation financière compris entre $2,1 \%$ pour le commerce de détail spécialisé et $3,87 \%$ pour le commerce de gros. Les durées moyennes de stage varient de un à trois jours. Globalement, l'ensemble des entreprises aborde positivement le changement d'attitude des salariés face à la formation. La réforme est plutôt bien perçue, sauf pour l'organisme de formation qui n'y voit aucun avantage. En revanche, l'ensemble des responsables de formation dénoncent la complexification du travail administratif et l'absence de budget pour le DIF. La gestion de la formation est pluri-annuelle sur deux à sept ans. Seule l'entreprise de prestations de services permet un cumul du DIF par anticipation pour toutes les catégories de personnel. La plupart des entreprises ne prévoient pas d'augmenter leur budget formation, sauf celles contraintes par des formations réglementaires. Le budget du DIF sera pris sur le budget du plan de formation, sauf pour l'entreprise du commerce de détail alimentaire qui a prévu un budget spécifique. Par exemple, le budget DIF est pris sur le budget du plan de formation à hauteur de :

- $10 \%$ pour les formations techniques à destination des formateurs et du public opérationnel pour l'entreprise de prestations de services ;

- $90 \%$ pour toutes les catégories et tous les publics pour l'entreprise de tourisme ;

- $5 \%$ pour l'entreprise d'intérim, comme prévu par l'accord de branche.

Les salariés ont été informés par les institutions représentatives du personnel et par les outils de communication internes (bulletins de paie, réunions, courrier, plaquettes, journal interne...). Par exemple, le responsable formation de l'entreprise de commerce de gros a réalisé un guide du salarié pour le DIF et mis en place une « hot line ».

Tableau 6

Profils socio-économiques des entreprises du scénario opportuniste

\begin{tabular}{|l|c|c|c|}
\hline \multicolumn{1}{|c|}{ Branches } & Début des demandes & $\begin{array}{c}\text { Nombre de } \\
\text { demandes } \\
\text { (\% salariés) }\end{array}$ & Réponses \\
\hline $\begin{array}{l}\text { Commerce de détail } \\
\text { spécialisé }\end{array}$ & 4 mai 2004 (26 mois) & $\begin{array}{c}19 \\
(0,2 \%)\end{array}$ & cœur de métier, motivation \\
\hline Commerce de gros & ler janvier 2005 (18 mois) & $\begin{array}{c}10 \\
(2 \%)\end{array}$ & cœur de métier \\
\hline $\begin{array}{l}\text { Organisme de } \\
\text { formation }\end{array}$ & 1er janvier 2005 (18 mois) & $\begin{array}{c}5 \\
(20 \%)\end{array}$ & positive \\
\hline Prestations de service & 1er juin 2005 (13 mois) & $\begin{array}{c}50 \\
(33 \%)\end{array}$ \\
\hline Intérim & ler juillet 2002 par accord & $\begin{array}{c}2705 \\
(0,48 \%)\end{array}$ \\
\hline Tourisme social & de branche (48 mois) & 16 & positive \\
\hline
\end{tabular}

Source : Nora Alleki, Catherine de Géry, administration du questionnaire sur les pratiques de formation, juillet-août 2006. 
Le nombre de demandes de DIF par entreprise est très variable. Les demandes sont à formuler auprès des responsables formation, de la direction des ressources humaines ou de la direction générale en fonction de la taille de l'entreprise, et cela à tout moment, sauf pour l'entreprise de commerce de détail spécialisé qui souhaite que les demandes soient réalisées lors de l'entretien professionnel.

La gestion du DIF se réalise hors temps de travail pour les entreprises du commerce de détail spécialisé, du commerce de gros, de l'intérim et du tourisme. Ceci est principalement justifié par le travail posté. Pour l'entreprise du commerce de détail alimentaire, la gestion du DIF se fait en mixant les deux avec les formations spécifiques à l'entreprise et réalisées pendant le temps de travail. Enfin, l'organisme de formation et l'entreprise de prestations de service réalisent leurs DIF pendant le temps de travail. Seules les entreprises du commerce de détail spécialisé et de la prestation de services ont initié les périodes de professionnalisation pour des cadres.

\section{Le scénario de résistance :} un contournement net de la réforme

Ce scénario semble être adopté par une entreprise de l'habillement. Il s'agit d'une entreprise de textile de 2000 salariés qui connaît des difficultés de recrutement sur son bassin d'emploi. Les objectifs affichés de la politique de formation sont l'accompagnement des évolutions organisationnelles, managériales et techniques. Son taux de participation financière est de $2,8 \%$ et sa durée moyenne de stage est de 8 ou 9 heures (hors formations diplômantes) et est comprise entre 150 et 450 heures pour les formations diplômantes qui représentent un tiers du volume des heures de formation. Les directions planifient leur politique de formation entre un et quatre ans. Le budget formation ne doit pas augmenter et le budget DIF sera pris sur le budget du plan de formation à hauteur de $50 \%$ pour les actions liées à l'évolution de l'emploi ou participant au maintien de l'emploi et de $100 \%$ pour les actions visant le développement des compétences (formations CAP et baccalauréat professionnel, en particulier). L'information dispensée aux salariés est annuelle (inscription du compteur du solde DIF sur le bulletin et entretien annuel d'évaluation). Les demandes de DIF sont acceptées depuis le $1^{\text {er }}$ mai 2005 (cumul à partir de janvier), par écrit et à n'importe quel moment. Au mois de juillet 2006, une quinzaine ont été émises et acceptées pour $0,75 \%$ des salariés. Les premières demandes réalisées à partir de juin 2005 concernaient uniquement les cadres. Les récentes demandes concernent à $80 \%$ des non-cadres. Le DIF est utilisé à $50 \%$ pour des bilans de compétences et pour des formations en bureautique et langues. Enfin, le DIF se réalise uniquement hors temps de travail et ne peut être cumulé par anticipation. Les périodes de professionnalisation n'ont pas été développées.

En synthèse, dans aucun des quatorze cas étudiés, la réforme ne semble avoir suscité une gestion prévisionnelle de la formation (hypothèse H2). Les entreprises n'ont pas pris conscience d'une nécessaire gestion organisationnelle de la formation et n'uti-lisent que rarement la possibilité d'anticiper le DIF sur six ans, particulièrement pour des formations diplômantes dans les secteurs ayant des besoins de développement des compétences. Toutefois, les firmes cherchent à maintenir leur budget constant, en accord avec l'hypothèse H1. Lorsque le plan de formation recouvre principalement des formations réglementaires obligatoires, le budget de formation augmente proportionnellement à leur coût. Les actions de formation initialement prévues dans le plan de formation sont proposées sous la forme de DIF aux salariés.

Conformément à l'hypothèse H3, le DIF a lieu pendant le temps de travail, sauf pour le travail posté qui reste en hors temps de travail dans les PME et en temps de travail dans les grandes entreprises.

Dans les entreprises étudiées, les demandes de DIF sont bien liées à la politique de communication interne, au dialogue social ambiant et au degré d'information des salariés, en accord avec l'hypothèse H4. L'accompagnement et l'information à disposition des salariés restent le fruit d'une investigation et ne sont pas clairement proposés aux salariés sans demande préalable. Certains salariés (élus, syndiqués ou ayant déjà bénéficié de formation) accéderont plus aisément à l'information ( $c f$. tableau 7). 
Résultats des illustrations de scénarios des pratiques de formation post-réforme 2004

\begin{tabular}{|c|c|}
\hline $\begin{array}{l}\text { 1 - Offensive } \\
\text { - Budget en augmentation (beaucoup de formations régle- } \\
\text { mentaires dans le plan de formation) ; } \\
\text { - Pas de gestion prévisionnelle de la formation, sauf dans } \\
\text { le transport aérien ; } \\
\text { - Gestion du DIF (droit individuel à la formation) prioritaire- } \\
\text { ment sur le temps de travail ; } \\
\text { - Degré d'information sur le DIF important. }\end{array}$ & $\begin{array}{l}3 \text { - Opportuniste } \\
\text { - Recherche d'un taux de participation financière } \\
\text { constant; } \\
\text { - Pas de gestion prévisionnelle de la formation, sauf pour } \\
\text { l'entreprise de prestations de services; } \\
\text { - Gestion du DIF prioritairement sur le temps de travail ; } \\
\text { - Degré d'information sur le DIF réalisé avec des outils de } \\
\text { communication plutôt individualisés. }\end{array}$ \\
\hline $\begin{array}{l}2 \text { - Cas par cas } \\
\text { - Recherche d'un taux de participation financière } \\
\text { constant; } \\
\text { - Pas de gestion prévisionnelle de la formation, sauf dans } \\
\text { l'entreprise de travaux publics; } \\
\text { - Gestion du DIF prioritairement sur le temps de travail, } \\
\text { sauf pour les équipementiers (hors temps de travail); } \\
\text { - Degré d'information sur le DIF réalisé sur les supports } \\
\text { existants. }\end{array}$ & $\begin{array}{l}4 \text { - Résistance } \\
\text { - Recherche d'un taux de participation financière } \\
\text { constant; } \\
\text { - Pas de gestion prévisionnelle de la formation ; } \\
\text { - Gestion du DIF prioritairement sur le temps de travail, } \\
\text { sauf pour les équipementiers (hors temps de travail) ; } \\
\text { - Degré d'information sur le DIF minimal. }\end{array}$ \\
\hline
\end{tabular}

Source : Nora Alleki, Catherine de Géry, administration du questionnaire sur les pratiques de formation, juillet-août 2006.

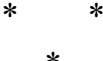

Pour l'instant, et avec le peu de recul dont nous disposons par rapport à la loi du 4 mai 2004, la réforme semble avoir peu d'impact sur les pratiques de formation. En effet, aucun investissement supplémentaire en formation n'est prévu du fait des opportunités supplémentaires de la réforme (contrat et périodes de professionnalisation, DIF). Par ailleurs, la politique de formation reste inscrite dans le court terme, le niveau privilégié des négociations demeure les branches professionnelles, et ce malgré la volonté affichée dans une partie de la loi du 4 mai 2004 de développer la négociation au sein de l'entreprise sur plusieurs aspects de la politique du travail et de l'emploi, dont la formation professionnelle continue. Les politiques de formation semblent ainsi structurellement prédéterminées, empêchant une régulation flexible au sein des firmes, particulièrement en raison de la place qu'occupe la branche dans la régulation du système, et de l'institu- tionnalisation du système. Déplacer le point d'ancrage de la branche à l'entreprise pourrait éventuellement faire sortir le système de formation professionnelle continue de son sentier de dépendance. Or, cela ne serait envisageable que pour les entreprises ayant accumulé suffisamment de savoirs et d'outils sur le fonctionnement du système ; elles seraient alors capables de déroger aux accords de branche et d'impulser une régulation d'entreprise autonome par rapport à la régulation de branche, institutionnelle et tripartite. Néanmoins, il est peu probable que la firme ne suive pas les objectifs de la branche parce que la branche, en formalisant les comportements, apporte des informations essentielles sur la concurrence.

Toutefois, la réforme datant du 4 mai 2004, il est encore trop tôt pour que les tendances évoquées puissent être stabilisées, d'autant qu'une nouvelle réforme de la formation continue est envisagée en matière de financement de la formation professionnelle continue et de transférabilité du droit individuel à la formation. 


\section{Bibliographie}

Alleki N. (2000), La flexibilisation du rapport salarial et la formation continue en France de 1971 à nos jours, thèse de doctorat de sciences économiques, université de Paris VII-Jussieu, Paris.

Alleki N. \& Géry C. de (2006), « Le droit individuel à la formation: un nouvel objet de gestion et de développement des compétences ? », Journée d'études CNAM, Usages sociaux de la notion de compétence : quels savoirs? quels individus?

Becker G.S (1964), Human capital : a theorical and empirical analysis, Chicago, University Chicago Press.

DARES (2006), La négociation collective en 2005, ministère du Travail, « Bilans et rapports ».

Doeringer P.B., Piore M.J. (1971), Internal labor market and manpower analysis, Lexington Books, Massachusetts.

Géry C. de (1997), Formation professionnelle continue, marché du travail et salariat, thèse de doctorat de sciences économiques, université de Picardie-Jules Verne, Amiens.

Grimshaw D. \& Rubery J. (1998), «Integrating the Internal and External Labour Markets », Cambridge Journal of Economics, 22.

Hanchane S. \& Stankiewicz F. (2004), « Approche organisationnelle de la formation: au-delà de la problématique beckerienne », Formation Emploi, $\mathrm{n}^{\circ} 85$, pp. $23-40$

d'Iribane Ph. (1989), La logique de l'honneur: gestion des entreprises et traditions nationales, Le Seuil.

Maggi-Germain N. (2004), « La formation professionnelle continue entre individualisation et personnalisation des droits des salariés", Colloque Les évolutions de la formation professionnelle continue, DARES, septembre.

Maurice M., Sellier F., Silvestre J.-J. (1982), Politique d'éducation et organisation industrielle en France et en Allemagne. Essai d'analyse sociétale, PUF, Paris.

Nelson RR \& Winter SG. (1982), An evolutionary theory of economic change, Belknap, Cambridge (Mass).

Rubery J., Wilkinson F. (1981), «Outwork and segmentation labor market ", in Wilkinson F. (Eds.), The Dynamics of Labour Market Segmentation, Academic Press.

Sengenberger W. (1981), « Labour market segmentation and the business cycle », in Wilkinson F. (Eds.), The Dynamics of Labour Market Segmentation, Academic Press.

Tallard M. (2005), « La formation tout au long de la vie », Regards sur l'actualité, n 309. 


\section{Résumé}

\section{Scénarios des pratiques de formation post-réforme de mai 2004}

Nora Alleki et Catherine de Géry

Cet article retrace les premiers impacts de la réforme du 4 mai 2004 sur les pratiques de formation des entreprises. À partir d'une typologie des pratiques de formation en quatre ensembles (offensive, opportuniste, au cas par cas et résistante) et en postulant l'inscription des pratiques de formation dans un sentier de dépendance, nous avons construit des scénarios des pratiques de formation postréforme que nous avons testés sur un échantillon de quatorze entreprises. L'article conclut que, pour l'instant, la réforme a peu d'impact sur les pratiques de formation.

\section{Mots clés}

Formation professionnelle en entreprise, Législation de la formation professionnelle, Typologie, Marché du travail

Journal of Economic Literature : L 53, M 53 